\title{
Strain Gradient Modification of the Mori-Tanaka Model to Predict the Elastic Properties of Layer by Layer (LBL) Manufactured Polymer/Clay Nanocomposites
}

\author{
Yaning $\mathrm{Li}^{1}$, Anthony M. Waas ${ }^{2}$ and Ellen M. Arruda ${ }^{3}$ \\ University of Michigan, Ann Arbor, MI, 48109
}

\begin{abstract}
A size-dependent strain gradient Mori-Tanaka model (SGMT) is developed by applying strain gradient elasticity to the classical Mori-Tanka (M-T) method. The SGMT model is shown to be able to accurately predict the experimentally observed increase in stiffness of a layer- by-layer (LBL)-assembled polyurethane-montmorillonite (PU-MTM) nanocomposite with various volume fractions. 3D Finite Element (FE) models of a representative volume element (RVE) are used to study the stiffness enhancement mechanism of the LBL PU-MTM nanocomposite. An effective constant thickness interphase is introduced in the FE simulations, which is analogous to using a formulation that incorporates strain gradient effects. An inverse method to determine the effective thickness and stiffness of the interphase layer using FE simulations is also presented.
\end{abstract}

\section{Nomenclature}

$\bar{\sigma}_{i j}, \bar{\varepsilon}_{i j}=$ applied constant stress and overall strain at the boundary

$\tilde{\sigma}_{i j}, \tilde{\varepsilon}_{i j}=$ perturbation stress and strain in the matrix

$\sigma_{i j}^{p t}, \varepsilon_{i j}^{p t}=$ second perturbation stress and strain in the inclusion

$C_{i j k l}^{0}, C_{i j k l}^{1}=$ stiffnesses of the matrix and the inclusion

$\varepsilon_{k l}^{0}, \varepsilon_{m n}^{*}=$ initial uniform strain in the matrix and the eigen strain

$S_{k l m n} \quad=$ Eshelby tensor

$\langle\bullet\rangle=$ spatial average of a field quantity

$E_{0}, E_{1}=$ Young's moduli of the PU matrix and the clay particle

$E_{x y}, E_{y x} \quad=$ in-plane Young's moduli of the nanocomposite

$E_{x z}, E_{y z}=$ out-of-plane Young's moduli of the nanocomposite

$v_{0}, v_{1}=$ Poison ratios of the PU matrix and the clay particle

$v_{x y}, v_{y x}=$ in-plane Poisson ratios of the nanocomposite

$v_{x z}, v_{y z}=$ out-of-plane Poisson ratios of the nanocomposite

$f \quad=$ volume fraction of clay

$l=\quad$ intrinsic material length scale

$\beta=$ dimensional parameter of strain gradient term

$a \quad=$ non-dimensional parameter of strain gradient term

\section{Introduction}

Classical two-phase micro-mechanics models, such as the Mori-Tanaka (M-T) model ${ }^{1,2}$ and the Halpin-Tsai (H$-\mathrm{T}$ ) model $^{3,4}$ fail to predict the mechanical stiffness of LBL PU-MTM nanocomposites, as reported by other researchers when applying these models directly to nanocomposite structures. ${ }^{5-8}$ One possible reason is that the

\footnotetext{
${ }^{1}$ Post-doc, Department of Mechanical Engineering, University of Michigan, Ann Arbor, MI, 48109.

2 Professor, Department of Aerospace Engineering, University of Michigan, Ann Arbor, MI, 48109.

${ }^{3}$ Professor, Department of Mechanical Engineering, University of Michigan, Ann Arbor, MI, 48109.
} 
classical two-phase M-T model does not include the contribution of the interphase, a finite zone of material that surrounds the inclusions and is a main structural feature of nanocomposites, significantly contributing to the property enhancement mechanisms of nanocomposites. However, the precise manner by which the interphase forms is still unknown. Depending on the components and the manufacturing processes of various types of nanocomposites, the existence of the interphase can be attributed to the gyration of the polymer chain, ${ }^{9,10}$ the effect of covalent cross-link density, ${ }^{11}$ or the thermodynamic effect. ${ }^{12-14}$ In the view of continuum mechanics, the interphase is a region that can be characterized as consisting of constrained polymer chains around nanoparticles. ${ }^{15}$ The presence of large strain gradients around the particles were shown by analytical and numerical models with perfect bonding. ${ }^{16,17}$ In a real nanocomposite, both the presence of an interphase and the existence of strain gradients can contribute to the stiffness enhancement mechanism and particle size effect simultaneously.

Currently, no reliable experimental method for determining the interphase properties exists, although many efforts have been made to find one. ${ }^{13,18,19}$ More information about the current status of the study of the role of the interphase in nanocomposite mechanics can be found in a review paper. ${ }^{20}$ In the present investigation, an inverse method using FE simulations of the nanostructure of the RVE is applied to calibrate the mechanical and physical properties of the interphase using tensile experimental data. By studying the strain gradient fields predicted by FE models without an interphase, the same order of interphase thickness can be obtained.

Classical elasticity assumes that the stress at a material point is uniquely determined by the strain at that point. Strain gradient elasticity which is a higher order continuum theory of elasticity (Micropolar or Cosserat theories ${ }^{21-26}$ ) argues that the stress at any particular point in a solid is not only determined by the strain at that point but also by the strains of neighboring points. If strain gradients are high, classical elasticity is not sufficiently accurate to describe the constitutive behavior of a material and we need to resort to strain gradient theory. In this investigation, the strain gradient M-T model (SGMT) is developed and is applied to predict the stiffness of the LBL PU-MTM specimens. An alternative strain gradient elasticity theory ${ }^{27,28}$ is introduced to modify the two-phase Mori-Tanaka model derived in the framework of classical elasticity. ${ }^{2}$ Excellent correlation with experimental data is obtained. Strain gradient plasticity has previously been applied to study the plastic behavior of PU-MTM nanocomposites. ${ }^{29}$

In addition, a serious drawback of the classical composite models is size-independency. The size effect of nanocomposites is such that for the same volume fraction of inclusions, if the inclusion size decreases, material properties such as strength and modulus dramatically increase. However, this size effect cannot be explained by conventional methods such as the M-T model and H-T model mentioned above, because these models have no material length scale included. Li et al. ${ }^{17}$ showed that by introducing an interphase to the M-T model, a close-form hierarchical multi-interphase model (HMM) can predict observed size effects. By introducing the strain gradient elasticity, the present SGMT model is also size dependent. The size-dependency of the SGMT model is explained conceptually in this study.

\section{Strain Gradient Mori-Tanaka Model}

In this section, a strain gradient Mori-Tanaka Model (SGMT) is derived by introducing the theory of strain gradient elasticity $^{27,28}$ to the classical Mori-Tanaka composite model. ${ }^{2}$

First, the constant stresses $\bar{\sigma}_{i j}$ are applied at the boundaries of pure matrix. The stiffness tensor of the matrix is $C_{i j k l}^{0}$.

The initial uniform strain in the matrix is $\varepsilon_{k l}^{0}$. The stress and strain in the matrix satisfy

$$
\bar{\sigma}_{i j}=C_{i j k l}^{0} \varepsilon_{k l}^{0} .
$$

If the inclusion is ellipsoidal, the stress and strain fields are always uniform and there is no strain gradient. Thus, the strain gradient elasticity degenerates into classical elasticity, and the classical composite theory holds. But, if the inclusion is non-ellipsoidal, the perturbation strain in the matrix is non-uniform. After adding the inclusions, the stress and strain fields in the matrix are not uniform and the uniformity depends on the value of the filler to matrix stiffness ratios (SRs) ${ }^{16}$.

The non-uniform perturbation stress and strain in the matrix are described using the strain gradient elasticity theory, ${ }^{27,28}$ as shown in Eq. (2). Thus, the average perturbation stress and strain relation becomes:

$$
\left\langle\tilde{\sigma}_{i j}\right\rangle=C_{i j k l}^{0}\left(\left\langle\widetilde{\varepsilon}_{k l}\right\rangle-\left\langle l^{2} \nabla^{2} \widetilde{\varepsilon}_{k l}\right\rangle\right) .
$$


where, the notation $\langle\bullet\rangle$ is used to represent the spatial average of a field quantity, $l$ is the intrinsic length scale of the material. For the LBL nanocomposites, $l$ is related with the thickness of the PU matrix layer. After perturbation, the averaged stress field in the matrix is

$$
\bar{\sigma}_{i j}+\left\langle\tilde{\sigma}_{i j}\right\rangle=C_{i j k l}^{0}\left(\varepsilon_{k l}^{0}+\left\langle\widetilde{\varepsilon}_{k l}\right\rangle-\left\langle l^{2} \nabla^{2} \widetilde{\varepsilon}_{k l}\right\rangle\right) .
$$

The strain filed in the inclusion is also perturbed. The perturbation strain in the inclusion is $\varepsilon_{k l}^{p t}$. For composites with very high SRs, such as the present PU-MTM nanocomposites, the overall strain in the inclusion are infinitely small. Therefore, the gradient of $\varepsilon_{k l}^{p t}$ can be neglected. Thus $\varepsilon_{k l}^{p t}$ is assumed uniform. After the perturbation, the averaged stress in the inclusion satisfies

$$
\bar{\sigma}_{i j}+\left\langle\widetilde{\sigma}_{i j}\right\rangle+\sigma_{i j}^{p t}=C_{i j k l}^{1}\left(\varepsilon_{k l}^{0}+\left\langle\widetilde{\varepsilon}_{k l}\right\rangle+\varepsilon_{k l}^{p t}\right)=C_{i j k l}^{0}\left(\varepsilon_{k l}^{0}+\left\langle\widetilde{\varepsilon}_{k l}\right\rangle-\left\langle l^{2} \nabla^{2} \widetilde{\varepsilon}_{k l}\right\rangle+\varepsilon_{k l}^{p t}-\varepsilon_{k l}^{*}\right),
$$

where, $C_{i j k l}^{1}$ is the stiffness of the inclusion and $\sigma_{i j}^{p t}$ is the second perturbation stress, and

$$
\varepsilon_{k l}^{p t}=S_{k l m n} \varepsilon_{m n}^{*}
$$

where, $S_{k l m n}$ is the Eshelby tensor and $\varepsilon_{m n}^{*}$ is the eigen strain ${ }^{2}$.

The total perturbation stress in both the matrix and the inclusion should be zero:

$$
\left\langle\tilde{\sigma}_{i j}\right\rangle+f \sigma_{i j}^{p t}=0
$$

where, $f$ is the volume fraction of the inclusion. The total spatial average strain of the composite is obtained by the rule of mixtures:

$$
\left\langle\bar{\varepsilon}_{k l}\right\rangle=\varepsilon_{k l}{ }^{0}+\left\langle\tilde{\varepsilon}_{k l}\right\rangle+f \varepsilon_{k l}^{p t}
$$

The strain gradient term is unknown. To initiate the study, a simplified assumption is made to the perturbation strain and strain gradient in the matrix:

$$
\left\langle\nabla^{2} \widetilde{\varepsilon}_{k l}\right\rangle=\beta\left\langle\widetilde{\varepsilon}_{k l}\right\rangle
$$

where, $\beta$ is a parameter relating the strain gradient to strain. Equation (2) and (8) yield

$$
\left\langle\tilde{\sigma}_{i j}\right\rangle=C_{i j k l}^{0}\left(a\left\langle\widetilde{\varepsilon}_{k l}\right\rangle\right)
$$

where,

$$
a=1-\beta l^{2} .
$$

Thus, the influence of the strain gradient term can be modeled by the non-dimensional strain gradient parameter $a$, and Eq. (4) becomes

$$
\bar{\sigma}_{i j}+\tilde{\sigma}_{i j}+\sigma_{i j}^{p t}=C_{i j k l}^{1}\left(\varepsilon_{k l}^{0}+\left\langle\widetilde{\varepsilon}_{k l}\right\rangle+\varepsilon_{k l}^{p t}\right)=C_{i j k l}^{0}\left(\varepsilon_{k l}^{0}+a\left\langle\widetilde{\varepsilon}_{k l}\right\rangle+\varepsilon_{k l}^{p t}-\varepsilon_{k l}^{*}\right) .
$$


The spatial average of the strain in the composite $\left\langle\bar{\varepsilon}_{k l}\right\rangle$ is linearly related to the constant applied stress $\bar{\sigma}_{i j}$ through the stiffness of the composite as:

$$
\bar{\sigma}_{i j}=C_{i j k l}\left\langle\bar{\varepsilon}_{k l}\right\rangle
$$

in which, the total spatial average strain is

$$
\left\langle\bar{\varepsilon}_{k l}\right\rangle=(1-f)\left(\varepsilon_{k l}^{0}+\left\langle\widetilde{\varepsilon}_{k l}\right\rangle\right)+f\left(\varepsilon_{k l}^{0}+\left\langle\widetilde{\varepsilon}_{k l}\right\rangle+\varepsilon_{k l}^{p t}\right) .
$$

Therefore, the components of the stiffness matrix can be solved from the system of equations above. Compared with the classical M-T model, an additional parameter $a$ describing the strain gradient effect is introduced.

\section{Application of the SGMT Model to LBL PU-MTM Nanocomposites}

Polymer/clay nanocomposites, as members of the organic-inorganic nanocomposite family, are promising materials exhibiting attractive hybrid physical and mechanical properties arising from synergistic effects among their components. ${ }^{17}$ The nanocomposites show improved mechanical properties over microcomposites with similar structures. No existing micro-mechanics models can predict the elastic properties of nanocomposites. In this Section, the SGMT model derived in Section II is applied to a LBL PU-MTM clay nanocomposite. ${ }^{30}$

\section{A. Nanostructure}

The LBL PU-MTM clay nanocomposite is a type of nanocomposite that results in a well-defined spacing between subsequent clay layers by virtue of the sequential layering of polymer and clay nanoparticles. ${ }^{31-32}$ The clay particles in the PU-MTM are exfoliated as layers composed of disk or flake-shaped particles dispersed in a PU matrix. Each clay layer is comprised of aligned clay flakes separated by the PU matrix in a manner similar to the brick and mortar structure of nacre. ${ }^{33,34}$ Specimens with various volume fractions of clay are obtained by changing the thickness of

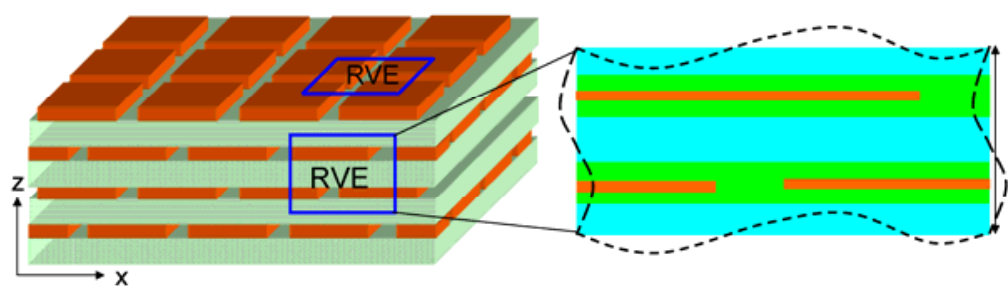

Figure1 Sketch of the idealized nanostructure of a LBL nanocomposite (Brown squares represent clay particles, green layers are polymer layers).

the polymer layers. Overall, these nanocomposites have a well-defined stratified nanostructure. The nanostructures of them are idealized by neglecting these irregularities and nonuniformities, as shown in Fig. 1. A RVE based on the nanostructure of the PU-MTM nanocomposite is chosen for Finite Element (FE) simulations and is also shown in Fig.1.

\section{B. Finite Element Simulations}

Both 2D plane strain and 3D FE models of the RVE shown in Fig. 1 are constructed in ABAQUS 6.6 for five specimens with various volume fractions: $5 \%, 7 \%, 9 \%, 12 \%$ and $20 \%$. The dimensions of the clay particles and the RVEs are determined from the nanostructure. The length of the clay particle is about $110 \mathrm{~nm}$, the thickness of clay layer is 3.6nm, the z-length of the RVE is $142 \mathrm{~nm}$, the x-length of the RVE varies and can be calculated for different volume fractions. ${ }^{17}$ The Young's modulus of the effective clay particle is $E_{I}=270 \mathrm{GPa}$, reported by Podsiadlo, et al., ${ }^{31}$ the Poisson's ratio is $v_{l}=0.375$; the Young's modulus of PU is $E_{0}=25 \mathrm{MPa}$, and its Poisson's ratio is $v_{0}=0.48$. Each phase is assumed to be linear elastic and isotropic. As a result of orthotropy, the modulus and Poisson's ratios of the 3D RVEs satisfy $E_{x y}=E_{y x}, v_{x y}=v_{y x}$, and $v_{y z}=v_{x z}$. By simulating the in-plane simple tension of 3D RVEs of various volume fractions, the elastic parameters $E_{x y}, v_{x y}$ and $v_{\mathrm{xz}}$ for various volume fractions can be obtained. In 2D plane strain RVEs, the modulus can be calculated by $E_{x y}=\left(1-v_{x y}^{2}\right) E_{x y}^{p s}$, where ' $p s^{\prime}$ ' represents plane strain, $v_{x y}$ is obtained from the results of 3D RVEs. Strain gradient effect is simulated by adding an interphase around the clay particles. The interphase is assumed to have the same Poisson's ratio as the PU matrix. To simulate the mechanical behavior of a nanocomposite specimen by a RVE that is far from its edge, periodic boundary conditions (PBCs) are 
used in the FE simulations. An approach of nodal constraint equations is used to impose PBCs in ABAQUS. Details of this approach can be found in the most recent literature. ${ }^{5,6,33}$

As an example, the 2D FE results of the tensile strain and stress distributions for a $5 \%$ clay specimen are shown in Fig. 2. It can be seen that the maximum strains are located in the matrix near the ends of clay particles and the maximum stresses are located in the middle of the clay particles. 3D FE results of the tensile strain distributions for $5 \%$ and $20 \%$ clay specimens are shown in Fig. 3.

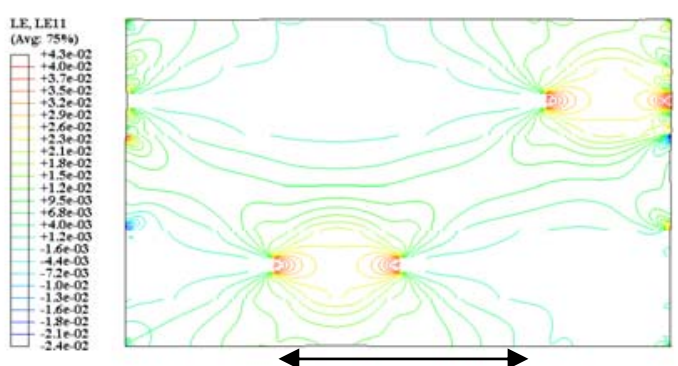

(a)

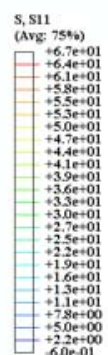

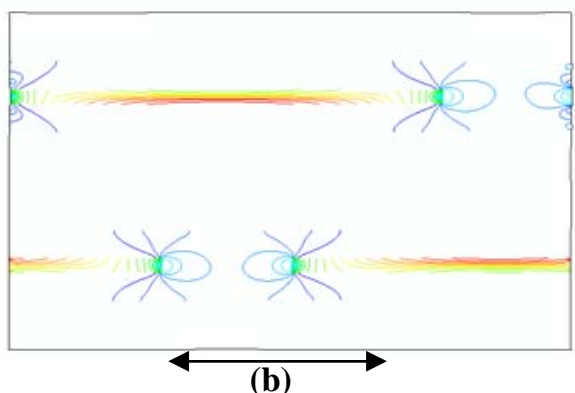

(b)

Figure 2 Contours of tensile strain and stress components from 2D FE results of $f=5 \%$ (the arrows represent the direction of tension). (a) Stain distribution. (b) Stress distribution.

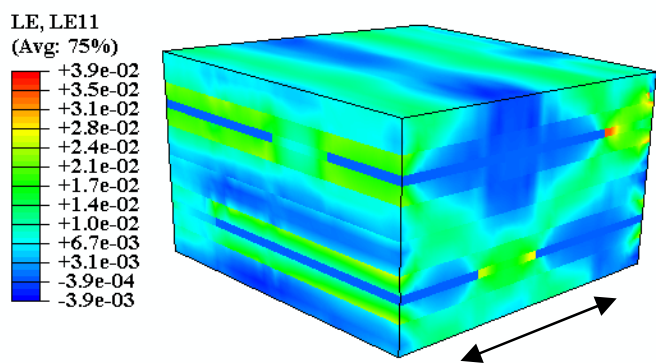

(a)

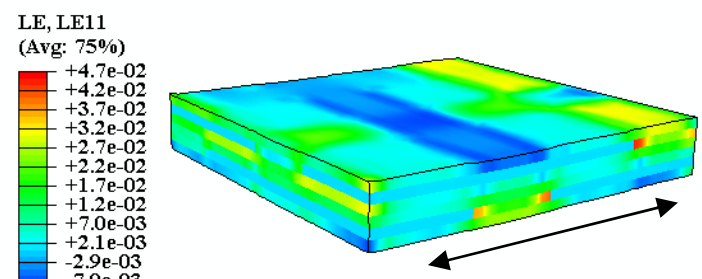

(b)

Figure 3 Contours of tensile strain from 3D FE results of $f=5 \%$ and $20 \%$ (the arrows represent the direction of tension). (a) $5 \%$. (b) $20 \%$.

The thickness and stiffness of the interphase will be determined from FE simulations and experimental results by an inverse method. The strategy of this inverse approach is that first, using the fact that a ductile to brittle transition is observed from the tests when the volume fraction increases to $12 \%$ (see Section IIIC), which indicates a contact or overlap of two neighboring interphase layers at $f=12 \%$, then the material transfer from three-phase to quasi twophase, and the thickness of the interphase is determined. This thickness equals half of the PU layer thickness of the $12 \%$ specimen. Then the stiffness of the interphase for the specimen with volume fraction $12 \%$ is obtained by comparing the stiffness predicted from the FE results and that which is measured from the tensile test.

\section{Ductile to Brittle Transition}

It is observed from tests that for small volume fractions of clay, the LBL PU-MTM nanocomposite shows a ductile stress-strain behavior, but when the volume fraction of clay increases to $12 \%$, a brittle material response occurs and the specimen breaks in the elastic regime. The same brittle behavior is displayed when the volume fraction is $20 \%$. Therefore, a ductile to brittle transition of this material occurs when the volume fraction of clay increases to about $12 \%$. This transition is captured by the 3D FE simulations with an interphase. Recording the maximum stresses in the 3D RVEs for various volume fractions at the same controlled tensile displacement, $1 \mathrm{~nm}$, when the corresponding overall strain is about $0.7 \%$, a jump of the maximum stress is shown

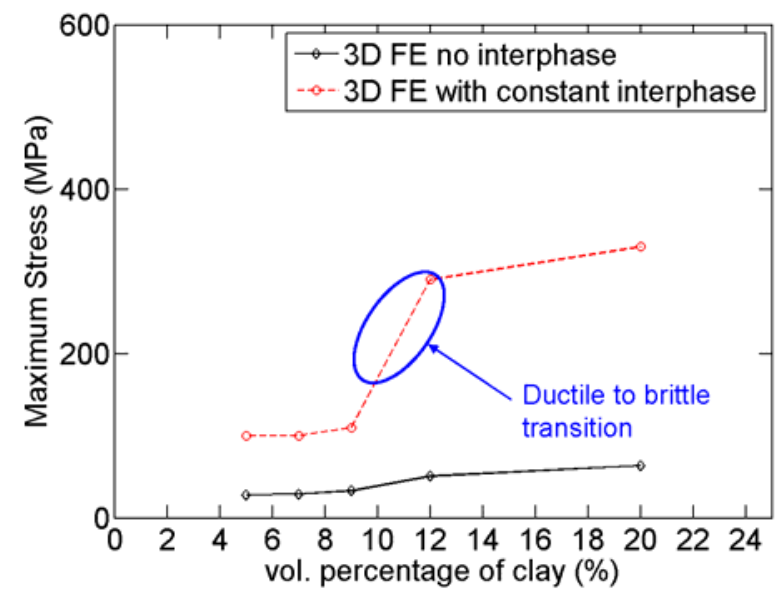

Figure 4 Ductile to brittle transition shown from FE results. 
in Fig.4, close to the case corresponding to $12 \%$, which is consistent with experimental observations. By comparing the FE simulations with and without an interphase, it can be concluded that this transition can mainly be attributed to the presence of an interphase. In addition, Fig. 4 shows that by introducing the interphase, the maximum stresses in the clay particles increase substantially for all volume fractions, indicating that the interphase plays an important role in transferring loads to clay particles and is therefore beneficial in strengthening the nanocomposite.

\section{Strain Gradient Effects from the FE results}

From the 2D FE results with no interphase, the distributions of tensile strain component along $\mathrm{z}$ axis (see Fig.1) in the PU matrix between two neighboring clay layers can be fitted by parabolas accurately. For strains along $\mathrm{x}$ axis (see Fig.1) is quasi-constant. Thus the strain Laplacian can be calculated from the parabola distribution of strains. The distribution of the strain gradient term along $\mathrm{z}$ is plotted in Fig. 5, which shows a rapid increase of the absolute value of the strain gradient term, when approaching the clay/PU interface for the case of $5 \%$. A similar phenomenon is observed for all volume fractions. The area with large absolute value of strain gradients near the interface can be equivalent to an effective interphase. It is also shown in Fig. 5 that with the decrease of volume fraction, the peaks of strain gradient term tends to

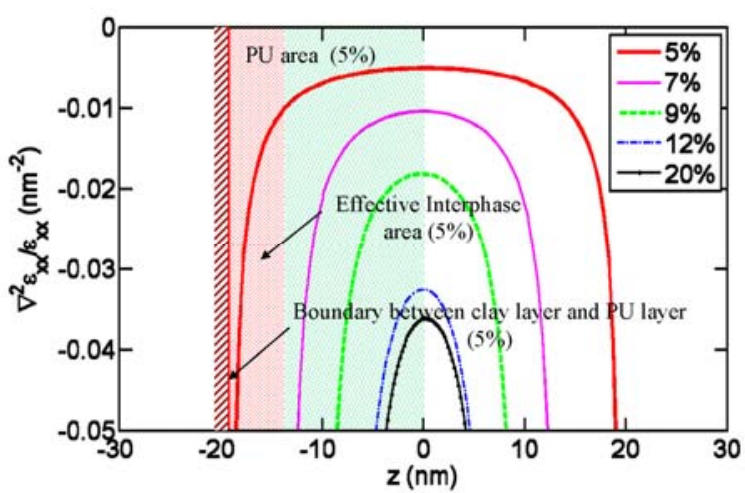

Figure 5 Distribution of strain gradients between neighboring clay layers for $2 \mathrm{D}$ RVEs with no interphase.

flatten, so the assumption of an effective interphase layer with constant thickness used in FE simulations is reasonable. The value of 7.2nm thickness for the interphase, calibrated in Section IB, is proved to be consistent with the strain gradient analysis, as shown in Figure 5, in which the thickness of the shaded effective interphase area is of the same order as that calibrated from FE simulations.

\section{E. Results and Discussion}

In this section, the SGMT model, 3D FE model, $\mathrm{H}-\mathrm{T}$ model, and the classical M-T model are all used to predict the Young's modulus of the PU-MTM specimens at various volume fractions. These predictions are compared with the experimental data reported by Kaushik et al. ${ }^{30}$, as shown in Fig. 6 .

Figure 6 shows that with the assumption of no interphase, all analytical and numerical model predictions are well below the experimental data. With no interphase, the $\mathrm{H}-\mathrm{T}$ over-predicts the FE results and the M-T model is consistent with the FE results for small volume fractions but under-predicts the FE results for larger volume fractions. 3D FE model with an interphase with constant thickness $7.2 \mathrm{~nm}$, and the stiffness $425 \mathrm{MPa}$ accurately predicts the stiffness of all specimens, spanning the volume fraction range 5\% to $20 \%$. By assuming the parameter $a$ in the strain gradient theory is linearly related to the volume fraction $f: a=a_{0}+H f_{p}$, and, by taking $a_{0}=0.07$, and $H=-0.17$, the SGMT model captures the stiffness of all specimens accurately, as shown in Fig. 6.

$\mathrm{Li}$ et al. ${ }^{16}$ showed that by introducing a constant interphase to the M-T model, a close-form hierarchical multi-interphase model (HMM) can be derived that is size-denpendent. The HMM takes into account the

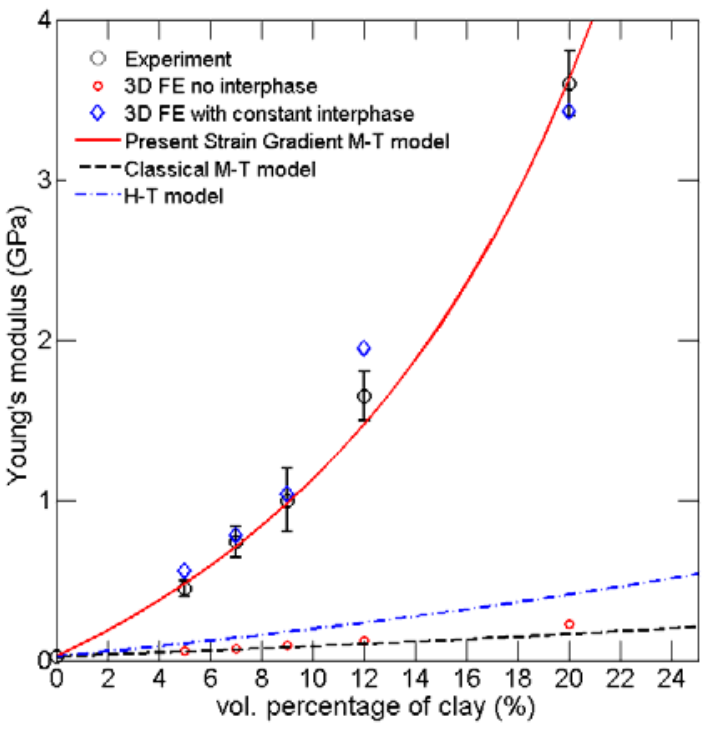

Figure 6 Comparison of prediction results of the SGMT model and other models with experimental data for LBL PU-MTM nanocomposites influence of an important size-dependent parameter: surface-to-volume ratio, and therefore can be used to predict 
particle size effects of nanocomposites. The spatial average of strain around the particles is related to a generalized surface-to-volume ratio implicitly, which indicates the SGMT is also particle size-dependant.

\section{Conclusion}

A strain gradient Mori-Tanaka (SGMT) model is developed by introducing the second strain gradient elasticity theory to the M-T model. A simplification is made to estimate the strain gradient effect by relating strain gradients to the strain through a non-dimensional parameter $a$. By assuming that $a$ is linearly related to the volume fraction of clay, the SGMT model can capture all the experimental data accurately. The size-dependency of the SGMT model is addressed conceptually. An analogy between the strain gradient effect and a constant interphase to account for the synergistic effect and size effect of nanocomposites is presented.

It is shown that strain gradient effect and/or the interphase effect is critical to understanding and evaluating the stiffness enhancement efficiency of nanocomposites, from a continuum mechanics modeling view point. Both approaches are applied to a LBL polyurethane-montmorillonite (PU-MTM) clay nanocomposite. The nanostructure of the LBL PU/MTM is analyzed. Both 2D and 3D FE models of RVEs are developed. An inverse method to determine the interphase thickness and its stiffness using 2D plane strain and 3D Finite Element simulations of the nanostructures and the experimental data is illustrated. The interphase thickness obtained from the strain gradient analysis of the 2D FE results is of the same order as that predicted by the inverse method.

\section{Acknowledgments}

The authors are grateful for financial sponsorship from the Office of Naval Research.

\section{References}

${ }^{1}$ Mori, T. and Tanaka, K., “Average Stress in Matrix and Average Elastic Energy of Materials with Misfitting Inclusions,” Acta Metall Mater, Vol. 21, 1973, pp. 571-574.

${ }^{2}$ Tandon, G. P. and Weng, G. J., "The Effect of Aspect Ratio of Inclusions on the Elastic Properties of Unidirectionally Aligned Composites,” Polymer Composites, Vol. 5, No. 11, 1984, pp. 327-333.

${ }^{3}$ Halpin, J. C., "Stiffness and Expansion Estimates for Oriented Short Fiber Composites," Journal of Composite Materials, Vol. 3, 1969, pp. 732-734.

${ }^{4}$ Halpin, J. C. and Kardos, J. L., “The Halpin-Tsai Equations: A review,” Polymer Engineering Science, Vol. 16, No. 5, 1976, pp. 344-352.

${ }^{5}$ Sheng N., Boyce M. C., Parks, D. M., Rutledge, G. C., Abes, J. I. and Cohen, R. E., "Multiscale Micromechanical Modeling of Polymer/clay Nanocomposites and the Effective Clay Particle," Polymer, Vol. 45, 2004, pp. 487-506.

${ }^{6}$ Hbaieb, K., Wang, Q. X., Chia, Y. H. J. and Cotterell, B., “Modelling Stiffness of Polymer/clay Nanocomposite,” Polymer, Vol. 48, 2007, pp. 901-909.

${ }^{7}$ Liu, H, and Brinson, L. C., “A Hybrid Numerical-Analytical Method for Modeling the Viscoelastic Properties of Polymer Nanocomposites,” Transactions of the ASME, Vol. 73, 2006, pp. 758-768.

${ }^{8} \mathrm{Li}$, Y., Waas, A.M., Arruda, E.M., “A particle size-shape-dependent three-phase two-step mori-tanaka method for Studying of the interphase and particle size and shape effects of of polymer/clay nanocomposites," ASME International Mechanical Engineering Congress \& Exposition (IMECE), Vol. 13, Boston, 2008, pp. 225-232.

${ }^{9}$ Fossey, S. "Atomistic modeling of polymer matrices in nanocomposites," Nanocomposites 2002: delivering new value to polymers conference proceedings, San Diego, CA, Executive Conference Management, 2002.

${ }^{10}$ Baschnagel, J. and Binder, K., "On the Influence of Hard Walls on Structural Properties in Polymer Glass Simulation," Macromolecules, Vol. 28, 1995, pp. 6808-6018.

${ }^{11}$ Putz K. W., Palmeri, M. J., Cohn, R. B., Andrews, R., and Brinson, C. L., "Effect of Cross-link Density on Interphase Creation in Polymer Nanocomposites,” Macromolecules, Vol. 41, 2008, pp. 6752-6756.

${ }^{12}$ Lipatov, Y. S. and Nesterov, A.E., Thermodynamics of Polymer Blends,(Polymer Thermodynamics Library, Vol 1), $1^{\mathrm{st}}$, Technomic Publishing, 1997.

${ }^{13}$ Ginzburg, V.V., Balazs, A.C., “Calculating Phase Diagrams of Polymer-platelet Mixtures Using Density Functional Theory: Implications for Polymer/clay Composites,” Macromolecules, Vol. 32, 1999, pp. 5681-5688.

${ }^{14}$ Helfand, E. and Tagami, Y. "Theory of the Interface between Immiscible Polymers. II," Journal of Chemistry and Physics, Vol. 56, 1972, pp. 3592-3601.

${ }^{15}$ Yung K. C., Wang J. and Yue, T. M., “Modeling Young’s Modulus of Polymer-layered Silicate Nanocomposites Using a Modified Halpin-Tsai Micromechanical Model,” Journal of Reinforced Plastics and Composites, Vol. 25, 2006, pp. 847-860.

${ }^{16} \mathrm{Li}$, Y., Waas, A. M., Arruda, E. M., “A Closed-form, Hierarchical, Multi-Interphase Composite Model-Derivation, Verification and application,” Journal of Mechanics and Physics of Solids (submitted), 2009.

${ }^{17} \mathrm{Li}$, Y., Waas, A. M., Arruda, E. M., 2010, “The Effects of Interphase and Strain Gradient on the Elasticity of LBL Polymer/Clay Nanocomposites,” International Journal of Solids and Structures (submitted), 2010. 
${ }^{18}$ Liu, R.Y.F., Bernal-Lara, T. E. et. al., “Interphase materials by Forced Assembly of Glassy Polymers,” Macromolecules, Vol. 37, 2004, pp. 6972-6979.

${ }^{19}$ Ciprari, D., Jacob, K., and Tannenbaum, R., "Characterization of Polymer Nanocomposite Interphase and its Impact on Mechanical Properties,” Macromolecules, Vol. 39, 2006, pp. 6565-6573.

${ }^{20}$ Pukanszky, B., "Interfaces and Interphases in Multicomponent Materials: Past, Present, Future," European Polymer Journal, Vol. 41, 2005, pp. 645-662.

${ }^{21}$ Toupin, R. A., "Elastic Materials with Couple Stresses,” Arch. Ration. Mech. Anal., Vol. 11, 1962, pp. 385-414.

${ }^{22}$ Koiter, W. T., “Couple Stresses in the Theory of Elasticity, I and II.,” Proc. K. Ned. Akad. Wet., Vol. (B), No. 67, 1964, pp. 17-44.

${ }^{23}$ Mindlin, R. D., “Micro-Structure in Linear Elasticity,” Arch. Ration. Mech. Anal., Vol. 16, 1964, pp. 51-78.

${ }^{24}$ Mindlin, R. D., “Second Gradient of Strain and Surface Tension in Linear Elasticity,” Int. J. Solids Struct., Vol. 1, 1965, pp. 417-438.

${ }^{25}$ Lakes, R. S. and Benedict, R. L., “Noncentrosymmetry in Micropolar Elasticity,” Int. J. Engng Sci., Vol. 20, No. 10, 1982, pp. 1161-1167.

${ }^{26}$ Lakes, R. S., “Viscoelastic Behavior of Chiral Materials,” International Journal of Mechanical Sciences, 2001, pp. 1-11.

${ }^{27}$ Aifantis, E. C., "Strain Gradient Interpretation of Size Effects,” International Journal of Fracture, Vol. 95, 1999, pp. 299314.

${ }^{28}$ Askes, H., and Aifantis, E. C., "Gradient Elasticity Theories in Statics and Dynamic - a Unification of Approaches," International Journal of Fracture, Vol. 139, 2006, pp. 297-304.

${ }^{29} \mathrm{Li}$, Y., Waas, A. M., Arruda, E. M., “A Non-local Visco-plastic Model with Strain Gradient Effects and Interphase Effects for Simulating the Stiffness and Yield Strength of a Class of Polymer Nanocomposites," Proceeding of 2008 ASME International Mechanical Engineering Congress \& Exposition (IMECE), Vol. 13, Boston, 2008, pp. 1119-1126.

${ }^{30}$ Kaushik, A. K., Podsiadlo, P., Qin, M., Shaw, C. M., Waas, A. M., Kotov, N. A., Arruda, E. M., “The Role of Nanoparticle Layer Separation in the Finite Deformation Response of Layered Polyurethane-Clay Nanocomposites,” Macromolecules, Vol. 42, No. 17, 2009, pp. 6588-6955.

${ }^{31}$ Podsiadlo, P., Kaushik, A. K., Arruda, E. M., Waas, A. M., Shim, B. S., Xu, J., Nandivada H., Pumplin, B. G., Lahann J., Ramamoorthy A., and Kotov, N. A., “Ultrastrong and Stiff Layered Polymer Nanocomposites,” Science, Vol. 318, 2007, pp. 8083.

${ }^{32}$ Podsiadlo, P., Kaushik, A. K., Shim, B. S., Agarwal, A., Tang, Z., Waas, A. M., Arruda, E. M., and Kotov, N. A., “Can nature's Design be Improved upon High Strength, Transparent Nacre-like Nanocomposites with Double Network of Sacrificial Cross Links,” Journal of Physical Chemistry, Vol. B, 2008, pp. 14359-14363.

${ }^{33}$ Tang, H., Barthelat, F. and Espinosa, H. D., "An Elasto-Viscoplastic Interface Model for Investigating the Constitutive Behavior of Nacre,” Journal of the Mechanics and Physics of Solids, Vol. 25, 2007, pp. 1410-1438.

${ }^{34}$ Barthelat, F., Tahng, H., Zavattieri, P. D., Li, C.-M., and Espinosa, H. D., "On the Mechanics of Mother-of-pearl: A Key Feature in the Material Hierarchical Structure,” Journal of the Mechanics and Physics of Solids, Vol. 55, 2007, pp. $306-337$. 